\title{
InAlAs/InGaAs/InP sub-micron HEMTs grown by CBE
}

\author{
G.O. Munns, M.E. Sherwin, T. Brock, G.I. Haddad, Y. Kwon, G.I. Ng and D. Pavlidis \\ Center for High Frequency Microelectronics, 1135 EECS Building, The Unicersity of Michigan. Ann Arbor, Michigan 48109-2122, USA
}

The InAlAs/InGaAs/InP high electron mobility transistor (HEMT) lattice matched to InP offers excellent high frequency, low noise operation for MMICs and low-noise amplifiers. The InP channel in the InP/InAlAs HEMT offers the advantages of improved high field velocity and higher breakdown voltages (the potential for higher power applications) over InGaAs channel HEMTs. InAlAs has been grown for the first time by CBE using TMAA producing InGaAs/InAlAs and InP/InAlAs HEMTs. Sub-micron InGaAs/InAlAs HEMTs with planar Si doping have been fabricated with $f_{1}$ values of $150 \mathrm{GHz}$ and $f_{\max }$ values of 160 $\mathrm{GHz}$. This device showed excellent pinch-off characteristics, with a maximum transconductance of $890 \mathrm{mS} / \mathrm{mm}$. The planar doped InGaAs channel HEMT had a higher $f_{\mathrm{t}}$ than a similar uniformly doped device. However, the non-optimized structure of the planar doped device resulted in a large output conductance of $120 \mathrm{mS} / \mathrm{mm}$, limiting $f_{\max }$ for that device. A sub-micron InP channel device was grown with a quantum well channel and double-sided planar Si doping. A sheet charge density of $4.4 \times 10^{12} \mathrm{~cm}^{-2}$ and associated room temperature mobility of $2800 \mathrm{~cm}^{2} / \mathrm{V} \cdot \mathrm{s}$ were achieved; however, the saturation current was low. The most likely causes for this are diffusion of the planar doping beneath the channel and the poor quality of the InP on InAlAs interface at the bottom of the quantum well channel.

\section{Introduction}

High electron mobility transistors (HEMTs) based in the InGaAs/InAlAs material system have demonstrated the best high frequency response of any transistor to date [1]. Offering high two-dimensional electron gas sheet densities, the added carrier confinement of a deep InGaAs channel, and relatively high electron mobility and peak drift velocity in the device channel, this system is well suited to sub-micron device work. Recently, InP/InAlAs HEMTs have been fabricated with excellent frequency characteristics utilizing the added advantages of yet higher peak drift velocities and significantly higher breakdown voltages [2] (i.e., higher power operation). Despite the potential of these material systems, few if any reports of InAlAs grown by CBE have been published. The advent of trimethyl amine alane (TMAA) with its low $\mathrm{O}_{2}$ sensitivity compared to tri-isobutyl aluminum or triethyl aluminum and its vapor pressure which is well suited to $\mathrm{CBE}$ has spurred development of AlGaAs with good results [3]. Chemical beam epitaxy offers an attractive method for growing these materials, i.e., the semi-infinite sources and the ability to utilize phosphorus, as in MOVPE, along with the molecular beam nature and monolayer interface abruptness of MBE. This is the first report of devices utilizing InAlAs grown by $\mathrm{CBE}$ with TMAA.

\section{Material growth}

The material for this study was grown in a first generation Varian (Intevac) CBE system using trimethyl indium (TMI), triethyl gallium (TEG), trimethyl amine alane, $100 \%$ arsine and phosphine. Conventional solid source silicon was used as an n-type dopant. Initial reports of CBE growth of aluminum containing compounds indicated that special precautions such as metal sealed mass flow controllers might be necessary due to oxygen permeation of any Viton seals [4]; however, all growths for this study have been conducted without modification to the gas delivery system. The adduct purified organometallics (Air Products) are delivered using palladium cell purified hydrogen carrier gas while the arsine and phosphine 
(Phoenix Research) are delivered through original equipment, Viton sealed mass flow controllers. While standard precautions to ensure vacuum integrity have been made, no extraordinary measures have been taken.

Optimization of the properties of bulk InAlAs was the initial step in obtaining device quality material. This process will be reported in detail elsewhere while only a few general results will be cited herein. Lattice matched material $(\Delta a / a<5$ $\times 10^{-4}$ ) showing featureless surface morphology was grown within 3 growth runs after inital bubbler installation. Fig. 1 shows a double crystal $\mathrm{X}$-ray scan, typical of our lattice matched material, exhibiting a large number of Pendellosung oscillations. The ease with which good quality material was obtained is primarily attributable to a wide growth window in parameter space. Unintentionally doped layers were n-type with $300 \mathrm{~K}$ apparent background carrier concentrations of $\sim 2 \times 10^{14} \mathrm{~cm}^{-3}$ and a mobility of $\sim 1000$ $\mathrm{cm}^{2} / \mathrm{V} \cdot \mathrm{s}$ for a thick layer grown directly on semi-insulating InP using no underlying buffer. This comapares favorably to earlier published reports of OMVPE [2,5] and MBE [6]. Secondary ion mass spectrometry (SIMS) indicates that the material is probably compensated showing Si levels near $1 \times 10^{17} \mathrm{~cm}^{-3}$, oxygen levels less than

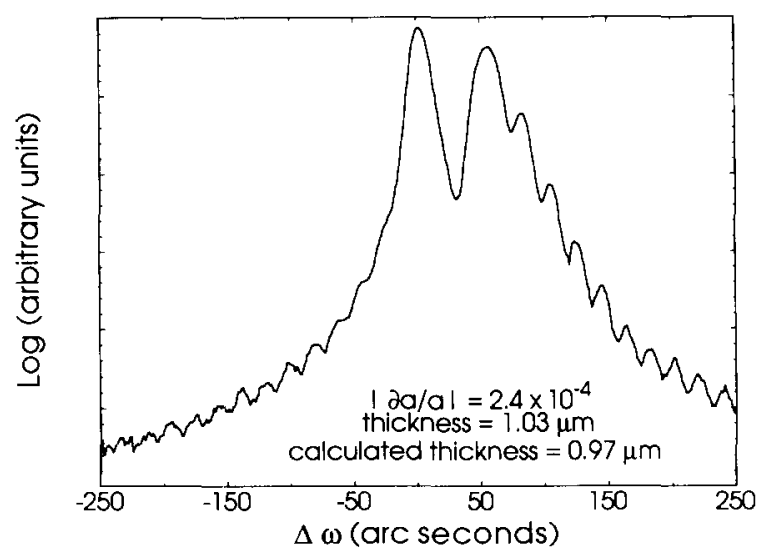

Fig. 1. Double crystal X-ray scan of a typical InAlAs layer grown directly on InP substrate. Multiple pendellösung oscillation indicates high quality.

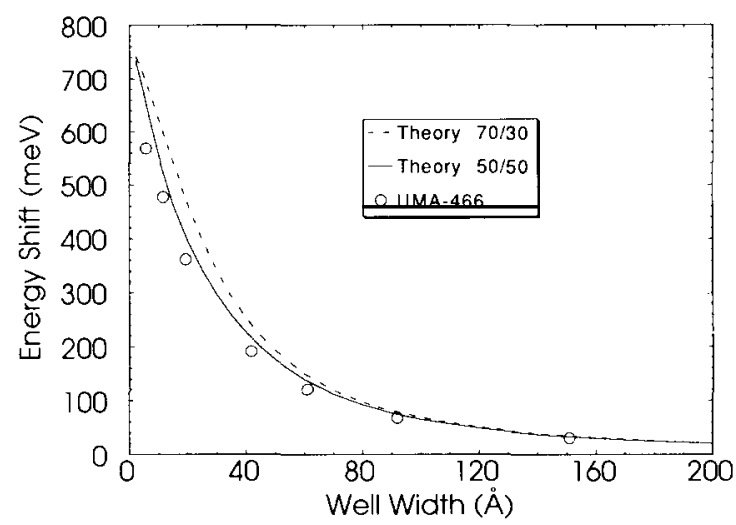

Fig. 2. Low temperature ( $15 \mathrm{~K})$ photoluminescence energy shift relative to bulk InGaAs of InGaAs/InAlAs uncoupled single quantum wells of various thickness. The thickness of each well was extrapolated from bulk growth rates.

$2 \times 10^{18} \mathrm{~cm}^{-3}$, and carbon levels near detection limit of about $2 \times 10^{16} \mathrm{~cm}^{-3}$. Parametric analysis of a SIMS study indicates that the probable source of the oxygen and silicon contamination is the hydrides, while the carbon clearly stems from the organometallics. Low temperature $(15 \mathrm{~K})$ photoluminescence is single peaked with a FWHM of $18.5 \mathrm{meV}$.

A series of uncoupled quantum wells was grown to investigate the growth of heterostructures, see fig. 2. The lack of good agreement between theory (for both $70 / 30$ and 50/50 conduction/valence band discontinuity) and experiment at narrow well widths is believed to be due to a transient flow increase just after flow is initiated, characteristic of the long vent/run lines of the first generation Varian CBE system. This results in initial growth rates greater than those at steady state, similar to flux transients in Knudsen cells found by Hafich et al. [7] and previously seen in InGaAs/InP quantum wells [8]. The final step was the calibration of the n-type dopant. It was found that the doping efficiency was approximately a factor of 5 lower than in InGaAs and InP grown under comparable conditions. These phenomena are also observed in conventional solid source MBE in our laboratory [9]. 


\section{Device fabrication}

Non-optimized InGaAs/InAlAs uniformly doped HEMTs were subsequently grown. Hall measurements of this uniformly doped structure yielded a mobility of $10,250 \mathrm{~cm}^{2} / \mathrm{V} \cdot \mathrm{s}$ at room temperature $\left(43,000 \mathrm{~cm}^{2} / \mathrm{V} \cdot \mathrm{s}\right.$ at $\left.77 \mathrm{~K}\right)$ and a corresponding sheet carrier concentration (2DEG) of $\sim 2 \times 10^{12} \mathrm{~cm}^{-2}$. Subsequently, a planar doped InGaAs/InAlAs HEMT with mobility of $7000 \mathrm{~cm}^{2} / \mathrm{V} \cdot \mathrm{s}\left(16,000 \mathrm{~cm}^{2} / \mathrm{V} \cdot \mathrm{s}\right)$ and corresponding $2 \mathrm{DEG}$ of $3.8 \times 10^{12} \mathrm{~cm}^{-2}$ was grown. In addition, an InAlAs/InP double sided planar doped HEMT with $2800 \mathrm{~cm}^{2} / \mathrm{V} \cdot \mathrm{s}\left(6300 \mathrm{~cm}^{2} / \mathrm{V}\right.$. s) and 2DEG of $4.4 \times 10^{12} \mathrm{~cm}^{-2}$ was grown. Pulse doped samples showed enhanced silicon incorporation/activation efficiency by a factor of about 3. Table 1 shows the results of Hall measurements on these and several other layers which were not fabricated into devices. Fig. 3 shows a typical device epitaxial structure for the pulse doped samples.

Discrete devices were subsequently fabricated using a hybrid optical/e-beam lithography process. Mesas were formed using a wet chemical etch $\left(\mathrm{H}_{3} \mathrm{PO}_{4}: \mathrm{H}_{2} \mathrm{O}_{2}: \mathrm{H}_{2} \mathrm{O}\right)$. An image reversal process was subsequently employed to define the ohmic $(\mathrm{Ge} / \mathrm{Au} / \mathrm{Ni} / \mathrm{Ti} / \mathrm{Au})$ patterns with a 2 $\mu \mathrm{m}$ source drain spacing. Rapid thermal annealing was used to alloy the contacts. The $0.2 \mu \mathrm{m}$ gate level lithography was performed using bilayer electron beam resist technology which employed a side lobe exposure technique. The critical gate recessing was done after development of the e-beam resist using a citric-acid : $\mathrm{H}_{2} \mathrm{O}_{2}: \mathrm{H}_{2} \mathrm{O}$

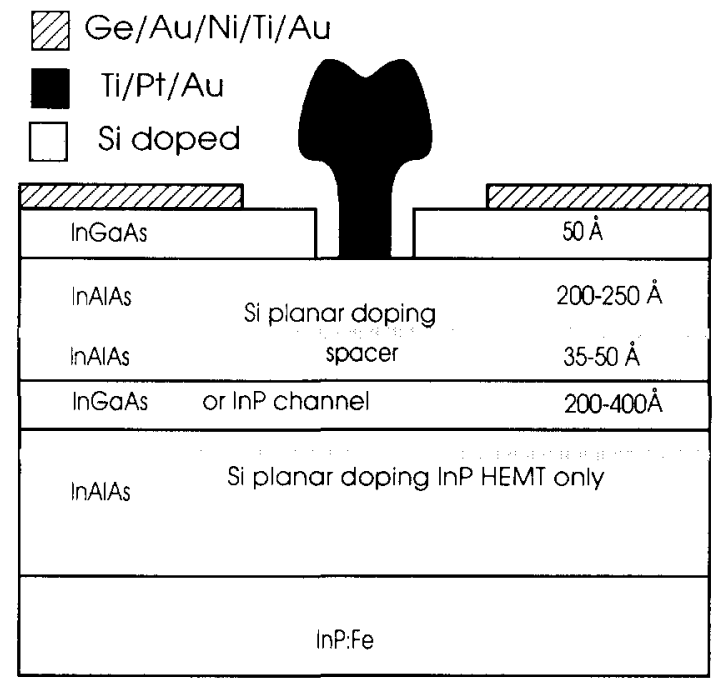

Fig. 3. Schematic cross-section of typical pulse doped HEMT epilayer structure.

etchant or a $\mathrm{H}_{3} \mathrm{PO}_{4}: \mathrm{H}_{2} \mathrm{O}_{2}: \mathrm{H}_{2} \mathrm{O}$ etchant. The gate metallization was $\mathrm{Ti} / \mathrm{Pt} / \mathrm{Au}$ and was lifted off with acetone.

\section{Device performance}

Table 1 summarizes the DC and microwave results for InGaAs/InAlAs HEMTs, uniformly doped and with planar doping, as well as the InP/InAlAs double sided planar doped HEMT. Microwave characterization of the devices was done from 0.5 to $26.5 \mathrm{GHz}$ with an HP8510 network analyzer and a Cascade prober. Microwave devices consisted of a two finger gate

Table 1

DC and microwave performance for the various structures

\begin{tabular}{llllcll}
\hline Structure & $\begin{array}{l}\mu \\
\left(\mathrm{cm}^{2} / \mathrm{V} \cdot \mathrm{s}\right)\end{array}$ & $\begin{array}{l}2 \mathrm{DEG} \\
\left(\mathrm{cm}^{2}\right)\end{array}$ & $\begin{array}{l}G_{\mathrm{m}} \\
(\mathrm{mS} / \mathrm{mm})\end{array}$ & $\begin{array}{l}G_{\mathrm{ds}} \\
(\mathrm{mS} / \mathrm{mm})\end{array}$ & $\begin{array}{l}f_{1} \\
(\mathrm{GHz})\end{array}$ & $\begin{array}{l}f_{\max } \\
(\mathrm{GHz})\end{array}$ \\
\hline InGaAs, ud & 10250 & $2.0 \times 10^{12}$ & 530 & 70 & 120 & 165 \\
InGaAs, pd & 7000 & $3.8 \times 10^{12}$ & 890 & 120 & 150 & 160 \\
InGaAs, pd & 8700 & $3 \times 10^{12}$ & & & & \\
InP, pd & 2800 & $4.4 \times 10^{12}$ & 200 & 31 & & 55 \\
InP, pd & 3850 & $2.2 \times 10^{12}$ & & & &
\end{tabular}

a) $\mathrm{ud}=$ uniformly doped; $\mathrm{pd}=$ planar doped. 

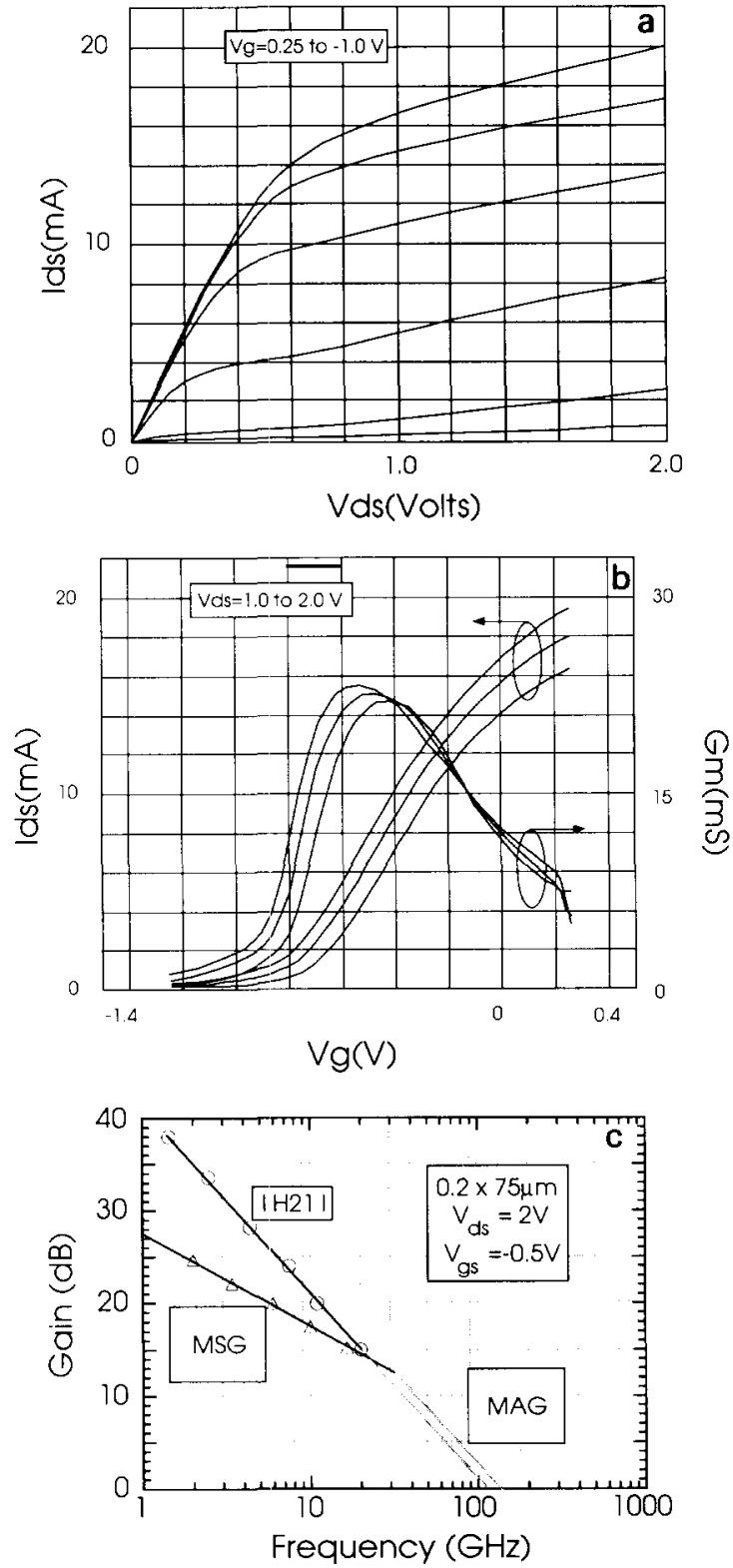

Fig. 4. Electrical characteristics from uniformly doped In GaAs/InAlAs HEMT: (a) $I_{\mathrm{ds}}-V_{\mathrm{ds}}$ characteristics of a $0.2 \times 45$ $\mu \mathrm{m}$ device; (b) transfer characteristics $\left(g_{\mathrm{m}}-V_{\mathrm{gs}}\right.$ and $\left.I_{\mathrm{ds}}-V_{\mathrm{gs}}\right)$ of $0.2 \times 45 \mu \mathrm{m}$ device; (c) current gain $\left(\left|H_{21}\right|\right)$ and power gain versus frequency for $0.2 \times 75 \mu \mathrm{m}$ device.

arranged in a pi configuration. Fig. 4 shows typical DC characteristics for a uniformly doped InGaAs/InAlAs device. Both InGaAs/InAlAs devices showed excellent pinch-off characteristics, with the planar doped device showing a slightly larger output conductance, which resulted in a depressed $f_{\max }$. All of the InGaAs/InAlAs devices showed very low gate leakage, typically lower than $12.5 \mu \mathrm{A}$ at $-1 \mathrm{~V}$. The breakdown voltage for the InGaAs/InAlAs devices was approximately $2.5 \mathrm{~V}$. The high transconductance of the planar doped sample is primarily due to two factors: an increased saturation current $(50 \mathrm{~mA}$ versus $17 \mathrm{~mA}$ at $V_{\mathrm{g}}=0 \mathrm{~V}$ ), and the placement of the gate closer to the channel as a result of the layer structure.

The InAlAs/InP channel devices had breakdown voltages of $4.5 \mathrm{~V}$. The devices showed low saturation currents $(10-15 \mathrm{~mA})$ and low transconductances $(\sim 200 \mathrm{mS} / \mathrm{mm})$. Possible explanations include arsenic contamination of the quantum well channel and diffusion of the planar doping due to non-optimized substrate temperature. We have previously observed arsenic contamination when InP is grown on top of arsenic containing layers [8]. The gate was very leaky, indicating that the gate metal was placed too close to the upper planar doping region. A single sided HEMT with a thick InP channel has been grown and it shows $300 \mathrm{~K}$ mobility of 3850 $\mathrm{cm}^{2} / \mathrm{V} \cdot \mathrm{s}$ with a $2 \mathrm{DEG}$ of $2.2 \times 10^{12} \mathrm{~cm}^{2}$, which is superior to other quantum well channel devices that we have grown with comparable sheet charge densities.

\section{Conclusions}

The first InAlAs containing devices grown by CBE using TMAA have been reported. InAlAs bulk layers show background carrier concentrations of $2 \times 10^{14} \mathrm{~cm}^{-3}$, with $15 \mathrm{~K}$ photoluminescence FWHM of only $18.5 \mathrm{meV}$. Planar doped InAlAs/InGaAs HEMTs, grown by CBE, have shown $f_{\mathrm{t}}$ values of $150 \mathrm{GHz}$ and $f_{\max }$ values of $160 \mathrm{GHz}$. Significant improvement in quality and device performance should be realized by eliminating contaminates from the hydride lines by using drying filters, by optimization of the structure and through improvement in the source purity of the TMAA (Air Products has already delivered improved source material). 


\section{Acknowledgments}

The authors would like to thank Mr. Marcel Tutt for his assistance with the device testing. This work was supported by the US Army Reseach Office, URI Program, Contract DAAL0386-K-0007.

\section{References}

[1] P.C. Chao, A.J. Tessmer, K.H.G. Duh, P. Ho, M.Y. Kao, P.M. Smith, J.M. Ballingall, S.M.J. Liu and A.A. Jabra, IEEE Electron Device Letters EDL-11 (1991) 59.
[2] L. Aina, M. Bergess, M. Mattingly, J.M. O'Connor, A. Meerschaert, M. Tong, A. Ketterson and I. Adesida, IEEE Electron Device Letters EDL-12 (1991) 483.

[3] C.R. Abernathy, A.S. Jordan, S.J. Pearton, W.S. Hobson, D.A. Bohling and G.T. Muhr, Appl. Phys. Letters 56 (1990) 2654

[4] Private conversation with Jim Cerny (Intevac).

[5] R. Bhat, M.A. Koza, K. Kash, S.J. Allen, W.P. Hong, S.A. Scharz, G.K. Chang and P. Lin, J. Crystal Growth 108 (1991) 441

[6] G.J. Davies, T. Kerr, C.G. Tuppen, B. Wakefield and D.A. Andrews, J. Vacuum Sci. Technol. B 2 (1984) 219.

[7] M.J. Hafich, H.Y. Lee, G.Y. Robinson, D. Li and N. Otsuka, J. Appl. Phys. 69 (1991) 752.

[8] M.E. Sherwin, F.L. Terry, G.O. Munns and G.I. Haddad, presented at TMS 5th Biannual Workshop on OMVPE, April 1991.

[9] Private conversation with Y.C. Chen (Univ. of Michigan). 